\title{
$\alpha$-L-Fucosidase in Cultured Skin Fibroblasts from Normal Subjects and Fucosidosis Patients
}

\author{
NICHOLAS G. BERATIS, (27) BRYAN M. TURNER. GUNDULA LABADIE, \\ AND KURT HIRSCHHIORN \\ The Division of Medical Genetics, Department of Pediatrics, Mount Sinai School of Medicine of the City. University \\ of New York, New York, USA
}

\begin{abstract}
Summary
(-L-Fucosidase (EC. 3.2.1.51) activity was studied in cultured skin fibroblasts obtained from 23 members of a family in which two cases of fucosidosis type 2 had occurred and in the fibroblasts of a patient with fucosidosis type 1 . Both the 4 methylumbelliferyl glycoside and the $p$-nitrophenyl derivative were used as substrates. pH activity profiles showed two major peaks of activity. With the fluorogenic substrate pII optimum was at 4.5, whereas with the colorigenic substrate maximum activity was at pH 5.7. No activity was found in the fibroblasts of the three patients with the colorimetric assay. With the fluorometric assay the mean activity in the two patients with fucosidosis type 2 was 4.1 and 2.8 (range 1.3-9.9); activity in the patient with fucosidosis type 1 was 4.6 mol 4 -methylumbelliferone/mg protein/hr (range 1.0-8.6). The specific activity in the lysates of the patients' fibroblasts decreased as the amount of cellular protein used per assay increased. Fucosidosis fibroblasts cultured for 3 and 5 days in medium without fetal calf serum showed almost the same levels of apparent residual activity as fibroblasts cultured in medium containing fetal calf serum. Maximum activity in the deficient fibroblasts was at pll 4.5-4.75. Mixing experiments between lysates of both types of fucosidosis and a normal fibroblast strain showed the expected enzymatic activities.
\end{abstract}

The mean $\alpha$-fucosidase activity in four heterozygotes for fucosidosis was 37.6 (range 24.1-48.7) and 30.3 (range 19.044.1) nmol final product split/mg protein/hr with the fluorogenic and the colorigenic substrate, respectively. In 12 normal fibroblast strains the mean activity \pm SD was $85.3 \pm 24.4$ (range 50.8-129.3) and 67.6 \pm 21.1 (range 31.1-118.3). However, in four family members in which the $\alpha$-L-fucosidase phenotype (by isoelectric focusing) was type 2-1, and who should therefore be carrying two normal alleles, the activity was within the heterozygote range. This indicates that occasional overlap between normal subjects and carriers may be present in cultured skin fibroblasts.

Increased specific activity of $\alpha$-L-fucosidase at pH 3.2-4.0 was observed after incubation of cell lysates with neuraminidase. The $\alpha$-L-fucosidase activity did not show any increase after fusion between fucosidosis fibroblasts types 1 and 2 .

\section{Speculation}

Although no biochemical evidence has been supplied indicating that the phenotypic differences between types 1 and 2 fucosidosis are genetically determined, it is possible that the two phenotypes are due to different mutations. The low residual activity found in the fibroblasts of both types of fucosidosis may represent either nonspecific hydrolysis of the fluorogenic substrate or genuine $\alpha$-L-fucosidase activity. The use of natural substrates and immunologic studies may clarify this problem.
Fucosidosis is a lysosomal storage discase resulting from severe deficiency of $\alpha$-L-fucosidase (EC. 3.2.1.51) (15). The enzymatic defect has been identified in various organs $(8,10,16)$, serum $(1,17)$, peripheral leukocytes $(1,3,10,11)$, and cultured skin fibroblasts $(10,18)$ of the patients. Traces of enzymatic activity have been demonstrated in cultured skin fibroblasts of two patients (18) whereas absence of activity was found in the fibroblasts of another case (10). Residual activity with altered physical properties has been found in the serum of two patients (4).

Based on clinical findings and family data we have identified two types of fucosidosis designated as types 1 and 2 (7). No biochemical differences have been demonstrated between the two types so far and, regardless of the phenotype, patients show, in various tissues, severe deficiency or total absence of $\alpha$-Lfucosidase activity.

In this paper we report the activity of $\alpha$-L-fucosidase in cultured skin fibroblasts obtained from 23 members of a family in which two cases of fucosidosis type 2 had occurred, and in the fibroblasts of an unrelated patient with fucosidosis type 1. Cells from both phenotypes of the disease have been studied and some of the properties of $\alpha$-L-fucosidase have been investigated.

\section{MATERIALS AND METHODS}

Skin biopsies were obtained from the inner surface of the forearm of two previously described patients with fucosidosis type 2 (7) and 21 other family members. Twelve fibroblast cultures were used as controls. RPMII 1640 medium supplemented with $16 \%$ fetal calf serum was used. (19). Skin fibroblasts from a patient with fucosidosis type 1 were kindly donated by Dr. A. L. Miller (20).

Skin fibroblasts were cultivated and prepared for enzyme determinations as previously described (2). Cells used in the study were between the fourth and eighth passage. Each cell preparation used for measurements of enzymatic activities was derived from two $75-\mathrm{cm}^{\prime \prime}$ culture flasks. At least two such preparations were studied from each culture.

$\alpha$-L-Fucosidase activity was measured by using 4-methylumbelliferyl-a-L-fucoside (21) as substrate. Assay conditions were the same as those reported earlier by us for peripheral leukocytes (1) except that the substrate concentration in the reaction mixture was $0.8 \mathrm{mM}$, the ply of the reaction was 4.5 , and the amount of cell protein used per assay was from $8-12 \mu \mathrm{g}$. Fluorescence readings were made in a Beckman model 772 ratio fluorometer at $450 \mathrm{~nm}$ after excitation at $360 \mathrm{~nm}$. $\alpha$-L-Fucosidase activity was also determined by using a modification of the colorimetric assay of Zielke et al. (18). The concentration of the substrate p-nitrophenyl- $\alpha$-L-fucoside (22) in the reaction mixture was increased to $3.0 \mathrm{mM}$ (at which concentration maximum velocity was obtained), the pll of the buffer was 5.7. and the incubation time was reduced to 2 hr. The amount of cellular 
protein used was $30-60 \mu \mathrm{g} / \mathrm{assay}$. $\alpha$-D-Mannosidase (EC. 3.2.1.24) activity was measured as described previously (1). $\beta$ D-Galactosidase (EC. 3.2.1.23) was estimated according to the method of 1 fo et al. (6). Protein was measured as reported by Lowry et al. (9). All assays were carried out in duplicate. Determinations for the establishment of the optimal assay conditions were performed in triplicate. Activity is expressed in nanomoles of 4-methylumbelliferone or nanomoles of $p$-nitrophenol released per mg protein per hr.

Neuraminidase treatment was performed by mixing cell lysates, neuraminidase from Vibrio cholera (23), and $10 \mathrm{mM}$ phosphate-citrate buffer, $\mathrm{pH} 4.0$, in a ratio of $4: 2: 1$. The neuraminidase used did not contain any preservatives. The mixture was incubated at $25^{\circ}$ for $18 \mathrm{hr}$.

Phenotyping of $\alpha$-L-fucosidase was performed by isoelectric focusing of peripheral leukocyte lysates in thin layer acrylamide gels, as described previously (14).

Cell fusion between fucosidosis fibroblasts of types 1 and 2 was accomplished with $\beta$-propiolactone-inactivated Sendai virus. Equal numbers of fibroblasts $\left(2 \times 10^{6}\right)$ from each strain suspended in $0.8 \mathrm{~m}$ l Hanks. balanced salt solution containing 25 mM Tricine buffer, pH 7.8 (19). Two-tenths milliliter of Sendai virus $(24)(4,000)$ HAU) were added to the fibroblasts and treated as previously described (5). Cells were harvested for enzymatic determinations 3 and 7 days after fusion.

\section{RESULTS}

The fluorescence released by the fibroblast lysates was linear from 1.2-32 $\mu \mathrm{g}$ cell protein/assay. No addition of albumin was necessary to linearize the relationship at low protein concentrations. The reaction was linear for at least $60 \mathrm{~min}$. The apparent residual activity present in the fucosidosis fibroblasts also increased linearly with time. Maximum velocity was obtained with a substrate concentration of $0.8 \mathrm{mM}$. pH activity curves with the fluorogenic substrate showed two major peaks and a major shoulder of enzyme activity. The highest of the two peaks was at pH 4.5. A number of smaller peaks and shoulders were also present, but their intensity and location varied in different lysates. When the colorigenic substrate was used the second peak was higher than the first one, with maximum at pll 5.7. Maximum activity in the cells of a patient with fucosidosis type 2 was at pH 4.5 to 4.75 (Fig. 1)

Figure 2 shows the family members whose cultured skin fibroblasts were studied. Table 1 lists the $\alpha$-L-fucosidase and $\alpha$-Dmannosidase activity in cultured skin fibroblasts. Subjects $/ 1-5$ and $I I-6$ were obligate heterozygotes, whereas $I I-l$ and $I I .+$ were identified reliably as carriers by demonstrating the segregation of

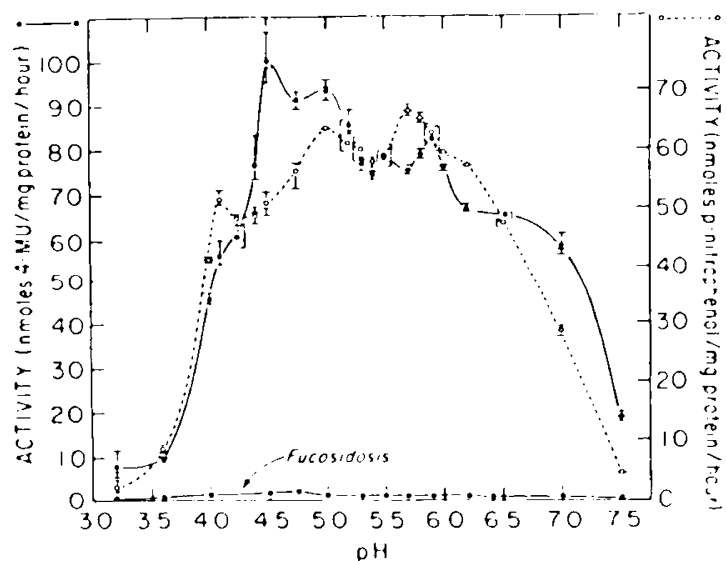

Fig. 1. phl activity profiles of $\alpha$-L-fucosidase from a normal skin fibroblast culture and a culture from a patient with fucosidosis type 2 . Both substrates, 4-methylumbelliferyl- $\alpha$-L-fucoside $(1-M U,-\longrightarrow)$ and $p$-nitrophenyl- $\alpha$-L-fucoside ( $p$-nitrophenol, O- - - O), were used.

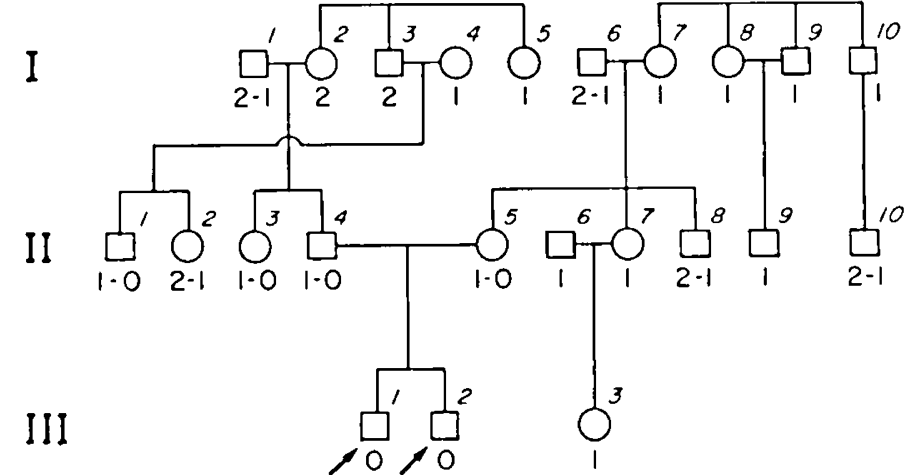

Fig. 2. The pedigree of the family. The phenotypes of $\alpha$-L-fucosidase are shown below each family member.

a silent allele in the family, as described previously (13). Patient $F-l$ was affected with fucosidosis type 1 . With the 4-methylumbelliferyl glycoside as substrate, the $(x$-L-fucosidase activity varied from $1.3-9.9$ in $I I l-1$, from $1.7-3.9$ in $I I I-2$, and from 1.0 8.6 in $F-1$. The specific activity in the lysates of the patients' fibroblasts decreased as the amount of the cellular protein used per assay increased. Figure 3 illustrates the fluorescence released and the apparent specific activity in a fibroblast lysate from subject $I I I-l$ in relationship to the amount of protein used per assay. Enzyme determinations performed on fresh intact fucosidosis cells and fresh mutant cells lysed with only one and three cycles of freezing and thawing did not show any increase in activity. The activity of $\alpha$-L-fucosidase in the fetal calf serum used for culturing was $153.6 \mathrm{nmol} 4$-methylumbelliferone $/ \mathrm{ml} / \mathrm{hr}$. In fucosidosis fibroblasts cultured in medium containing fetal calf serum the $\alpha$-L-fucosidase activity 3 and 5 days after early confluency was 3.1 and 2.0. In the same fibroblast strain, cultured in medium without fetal calf serum for 3 and 5 days, the activity was 4.1 and 1.5 , respectively. For all determinations the same amount of cellular protein was used per assay. $(\alpha-D-M$ - Mannosidase had a higher activity in the fibroblasts cultured in the absence of fetal calf serum. Mixing experiments between fibroblast lysates of both types of fucosidosis and the lysate of a normal fibroblast strain showed the expected enzymatic activities.

The activity of $\alpha$-L-fucosidase in cultured skin fibroblasts from the four heterozygotes for fucosidosis was generally lower than in the normal fibroblasts. No overlap occurred between carriers and normal subjects with the fluorogenic substrate when the mean activity of two cell preparations was considered. On single enzyme determinations occasional overlap between carriers and normal subjects was present.

Table 2 lists the $\alpha$-L-fucosidase and $\alpha$-D-mannosidase activity in all family members shown in Figure 2, except those included in Table 1. $\alpha$-L-Fucosidase activity in the family members $I-I, I$ $6, I I-8$, and $I I-I 0$ was found to be within the carrier range with both substrates; however, in all of these, the phenotype of $\alpha$-Lfucosidase was $2-1$. Clearly, such individuals must have two normal alleles and, therefore, cannot be carriers for fucosidosis. The activity was also within the heterozygote range in subjects 1 $2, I-3, I-7, I-8, I-I 0, I I-7$, and $I I I-3$.

$\alpha$-L-Fucosidase activity on the acidic side of a pll curve performed in a normal skin fibroblast lysate that was treated with neuraminidase was higher than in a fraction of the same lysate similarly treated with saline. At pH 4.5 the neuraminidasetreated sample had a slightly higher activity than the control, although the second peak had the same activity in both samples. No $\alpha$-L-fucosidase activity was found in the neuraminidase preparation. Three fractions from six normal and two fucosidosis fibroblast lysates were prepared. One fraction from each case was stored at $-80^{\circ}$ (fraction $A$ ) or treated with neuraminidase (fraction B) or treated with normal saline (fraction C). $\alpha$-L- 
Table 1 . Activity of $\alpha$-L-ficosidase and $\alpha$-D-mannosidase in cultured skin fibroblasts from homozygote's and heterozygotes for fucosidosis and normal subjects'

\begin{tabular}{|c|c|c|c|c|}
\hline \multirow[b]{2}{*}{ Subject } & \multirow{2}{*}{$\begin{array}{l}\text { Preparations } \\
\text { studied }\end{array}$} & \multicolumn{2}{|c|}{$\alpha$-L-Fucosidase } & \multirow[b]{2}{*}{ Mannosidase } \\
\hline & & 4-MUI: & $p$-NPF & \\
\hline Paticnts $(3)^{2}$ & 10 & 3.8 & () & 158.8 \\
\hline$I I I-I$ & 4 & 4.1 & 0 & 135.0 \\
\hline $111-2$ & 2 & 2.8 & 0 & 155.1 \\
\hline$F-1$ & 4 & 4.6 & () & 183.3 \\
\hline Heterozygotes (4) & 8 & 37.6 & 30.3 & 57.8 \\
\hline$I I .1$ & 2 & +7.9 & +4.1 & 56.4 \\
\hline $11-3$ & 2 & 48.7 & 31.7 & 46.7 \\
\hline $11-4$ & 2 & 24.1 & 26.6 & 44.9 \\
\hline 11.5 & 2 & 29.7 & 19.0 & 82.4 \\
\hline Normal (12) & 24 & $55.3 \pm 24.4^{3}$ & $67.6 \pm 27.1^{3}$ & $57.3 \pm 12.8^{3}$ \\
\hline Range & & $50.8-129.3$ & $31.1-118.3$ & $40.0-85.9$ \\
\hline
\end{tabular}

4-NUF: 4-methylumbelliferyl- $\alpha$-L-fucoside; $p$-NPF: $p$-nitrophenyl- $\alpha$-L-fucoside. Activity is expressed in nanomoles of final product split/mg protein/hr. Number of subjects in parentheses.

$2 I I I-I$ and $I I I-2$, patients with fucosidosis type $2 ; \%-l$, patient with fucosidosis $I y p d$.

${ }^{3}$ Mean $\pm \mathrm{SD}$.

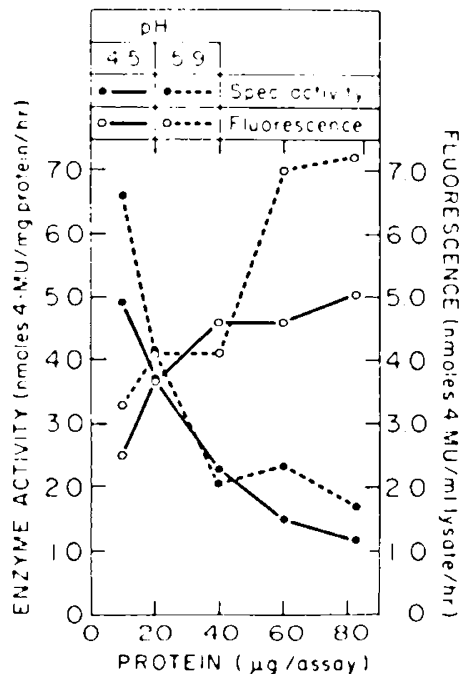

Fig. 3. Specific activity and fuorescence released versus the amount of protein used per assay from a fucosidosis type 2 skin fibroblast culture at pH 4.5 and 5.9. 4-MU: 4-methylumbelliferone.

Fucosidase determinations were performed at pH $3.6,4.5$, and 5.9 on all fractions. At pH 3.6 the enzyme activity in the neuraminidase-treated fraction $B$ of the normal fibroblasts was $36.3 \pm 9.6$, that is, significantly higher than the activity in the corresponding untreated fractions A $(15.3 \pm 3.0 ; t=5.183, P<$ $0.001)$ and $C(4.4 \pm 1.9 ; t=6.914, P<0.001)$. The difference between fractions $A$ and $C$ is also significant $(t=4.362, P<$ $0.0(1)$. At pH 4.5 the enzymatic activity in fraction $B(4+.6 \pm$ 8.2) was only slightly higher than that in fraction $C(40.0 \pm 6.4)$. This difference is not significant $(t=1.063, P<0.2)$. The activity, however, in fraction $A(74.6 \pm 18.6)$ was significantly higher than that of $\mathrm{B}(t=3.6(1), P<0.01)$ and $\mathrm{C}(t=4.362, P$, $<0.01)$. At $\mathrm{pH} 5.9$ the levels of the enzyme activity in fractions $B$ and $C$ were $34.0 \pm 7.6$ and $33.0 \pm 8.4(t=0.2(4 . P<0.5)$. respectively. In the fucosidosis lysates no difference among the three fractions was seen.

The activity of $x$-L-fucosidase did not show any increase after fusion between fucosidosis fibroblasts type 1 and 2 . Virustreated and mixed cells of the two types had the same levels of apparent enzyme activity as those observed in the control cultures. The percentage of binucleated cells in the virus-treated mixtures of fucosidosis type 1 and 2 fibroblasts was $9^{4} c$. The percentage of trinucleated cells was $0.5 \%$. In control cultures of
Table 2. $\alpha$-L-Fucosidase and $\alpha$-D-mannosidase activity in cultured skin fibroblasts from family me'mbers of pedigre'e shown in figure $3^{1}$

\begin{tabular}{|c|c|c|c|}
\hline \multirow{2}{*}{$\begin{array}{c}\text { Family mem- } \\
\text { ber }\end{array}$} & \multicolumn{2}{|c|}{$\alpha$-L-1 fucosidase } & \multirow[b]{2}{*}{$(x-1)-1$ annosidase } \\
\hline & L-MUF & $p-N P Y$ & \\
\hline$I-I$ & 29.6 & 31.5 & 32.0 \\
\hline $1.2^{2}$ & 27.2 & $26 . .3$ & +2.2 \\
\hline $1.3^{2}$ & 32.1 & $4(0.1$ & 60.8 \\
\hline 1.4 & 88.0 & 77.2 & 49.8 \\
\hline 1.5 & 114.8 & 72.2 & 60.2 \\
\hline $1-6$ & 38.8 & 19.7 & 93.5 \\
\hline $1.7^{2}$ & 36.7 & 30.5 & 99.4 \\
\hline$I-\delta^{2}$ & 35.3 & 26.9 & 81.7 \\
\hline 1.9 & 68.9 & 67.9 & 67.3 \\
\hline $1-10^{2}$ & 19.6 & 17.2 & 105.0 \\
\hline 11.2 & 89.5 & 75.8 & 53.1 \\
\hline $11-6)$ & 92.2 & 82.4 & 64.9 \\
\hline $11.7=$ & 25.5 & 19.8 & 65.3 \\
\hline 11.9 & 48.0 & 19.7 & 66.8 \\
\hline 11.9 & 66.6 & 43.2 & 52.4 \\
\hline 11.10 & 42.4 & 29.3 & 134.0 \\
\hline $111.3^{2}$ & +6.9 & 34.1 & 94.5 \\
\hline
\end{tabular}

'4-MUV: 4-methylumbelliferyl-a-L-fucoside; $p$-NPF: $p$-nitrophenyl$\alpha$-L-fucoside. Activity is expressed in nanomoles of final product split/ $\mathrm{mg} \operatorname{protein} / \mathrm{hr}$.

2 Considered to be possible carriers for fucosidosis.

mixed cells from both types, binucleated cells comprised $2{ }^{\circ} \%$. Both types of fucosidosis fibroblasts were from male donors and no further attempt was made to determine the percentage of hybrids between types 1 and 2 .

\section{DISCUSSION}

The use of the fluorogenic substrate for the study of the enzyme $\alpha$-L-fucosidase in cultured skin fibroblasts significantly increased the sensitivity of the assay and provided a simpler method for measurement of enzyme activity in these cells. pll activity profiles showed two major peaks of activity with both substratles, but the specificities of these two enzymatic peaks were different towards the two substrates used. Ziclke ot al. (18). using the p-nitrophenyl fucoside substrate, have reported a single optimum between pit 5.5 and 6.2.

Both peaks of activity were affected in the fucosidosis cells. suggesting that in some manner they are controlled at a single 
genetic locus. It is not clear at the present time whether the ipparent residual activity present in the mutant cells is due to races of $\alpha$-L-fucosidase activity or whether it is the result of conspecific hydrolysis of the substrate. Increasing amounts of :ellular protein per assay caused a reduction in the apparent ipecific activity. This could be due to an inhibitor present in the jatients cells, but this possibility was not supported by the indings of the mixing experiments between lysates of fucosidesis and normal fibroblasts. The fact that the residual activity present $n$ fresh mutant fibroblasts was the same as the activity obtained $n$ stored cells suggests that the residual activity in the fucosidosis :ells is not attributable to a labile enzyme. Since the fetal calf jerum has $\alpha$-L-fucosidase activity, the residual activity present in the fucosidosis fibroblasts could be derived from this source. Ihis, however, was not supported from the levels of the apparsnt activity found in the deficient fibroblasts that were cultured in medium without fetal calf serum for up to 5 days, although retention of the calf enzyme in the lysosomes for a longer period of time, if this enzyme is picked up by the deficient fibroblasts, is possible. Moreover, apparent residual activity, also found in the peripheral leukocytes of fucosidosis patients (1), weighs against the possibility of an exogenous origin of the hydrolytic activity in the deficient fibroblasts.

The heterozygous fibroblasts showed generally lower levels of enzymatic activity than the normal fibroblasts, but the distinction between them was not always clear. Better separation between the two genotypes was obtained with 4 -methylumbelliferyl glycoside as substrate. Estimation of the mean activity of two enzyme determinations in two different fibroblast preparations, each one derived from two flasks, improved the ability of the method to identify the carriers.

In addition to the patients parents and subjects $I I-I$ and $I I-3$. who were reliably identified as carriers by phenotyping. seven other family members were considered as possible carriers according to the levels of $\alpha$-L-fucosidase activity in cultured skin fibroblasts. The same individuals were characterized as carriers by studying the enzyme activity in purified lymphocytes (1). Subjects $I-I, I-6, I I-8$, and $I I-I 0$ had normal enzyme activity in the lymphocytes, and, since the $\alpha$-L-fucosidase phenotype was 2-1, they should be carrying two normal alleles (one $F u^{1}$ and one $\left.F^{2}\right)$. However, all of these individuals showed enzyme activity within the heterozygote range. Although some normal fibroblasts showed activities well within the carrier range, considering the mean of two determinations, no heterozygote demonstrated activity above 49.0 and 45.0 with the fluorogenic and colorigenic substrate, respectively.

It is possible that the increased specific activity of $\alpha$-L-fucosidase observed at pH 3.2-4.0, after incubation with neuraminidase, was due to the removal of sialic acid residues from the enzyme molecule and the consequent activation of an isozyme with a more acidic pH optimum than the main isozyme. This is supported by the finding that $\alpha$-L-fucosidase in various tisstes consists of as many as six isozymes which change electrophoretic mobility after treatment with neuraminidase (12, 14).

No biochemical evidence has been supplied up to the present time, indicating that the phenotypic differences between types 1 and 2 are genetically determined. If we had found complenentation in hybrids between types 1 and 2, this would have indicated that the two phenotypes of fucosidosis are due to different mutations, probably at different loci. All pairs of siblings affected with fucosidosis that have been reported so far were concordant for type 1 or 2, but a modifying gene(s) could conceivably cause the phenotypic differences. If this is the case, then one would expect to find, in the future, affected siblings discordant for types 1 and 2. The ancestors of most of the patients with both types of fucosidosis have been traced to Southern Italy. In the (wo) siblings with fucosidosis type 2 studied here, the fucosidosis gene was passed on to the patients through their grandmothers $(1,13)$, who were of Southern Italian and Sicilian origin. If the two fucosidosis phenotypes are due to different mutations, then the relatively high frequency of two different mutations in Southern Italy could be due to a selective advantage of the heterozygotes in the area. Similar examples could be mentioned, such as the presence of different mutations causing thalassemia or the existence of different alleles at the glucose-(n-phosphate dehydrogenase locus in populations affected with malaria in the past.

\section{CONCI.USION}

Cultured skin fibroblasts from 23 members of a family in which two cases of fucosidosis type 2 had occurred and from a patient with fucosidosis type 1 were studied. No $\alpha$-L-fucosidase activity was found in the fibroblasts of either type of fucosidosis with the $p$-nitrophenyl glycoside substrate, although traces of apparent residual activity were present with the 4-methylumbelliferyl derivative. The residual activity in the mutant fibroblasts increased with the incubation time and had a $\mathrm{pH}$ optimum similar to that seen in normal cells. However, the relationship between specific enzyme activity and cellular protein was not linear in the deficient fibroblasts. No inhibitor wats demonstrated in the fucosidosis cells. Fibroblasts from heterozygotes for fucosidosis had activity generally lower than normal fibroblasts, but overlap between these two genotypes was present occasionally. Treatment of normal fibroblast lysates with neuraminidase caused an increase in the specific activity at pll 3.2-4.0. No complementation was demonstrated in fusion experiments between fibroblasts from fucosidosis types 1 and 2. These studies failed to show a biochemical difference between the two types of fucosidosis.

\section{RIFERENCLS AND NOTES}

1. Beratis, N. G.. Turner, B. M., and Hirschhorn, K.: Fucosidesis: Detection of the carrice state in peripheral blood leukocytes. J. Pediat., 87: 1193 (1975).

2. Beratis, N. G., Turner, B. M. Weiss, R., and Hirschhorn, K.: Arblsulfatase B deficiency in Maroteaux-Lamy syndrome: Cellular studies and carrier identification. Pediat. Res.. 9: 475 (1975).

3. Borrone, C., Gatti, R., Trias, X., and Durand, P.: Fucosidosis: Clinical, biochemical, immunologic, and genetic studies in two new cases. J. Pediat., 84: $727(1974)$.

4. Di Mateo, G., Durand, P., Gatti, R., Maresca, A., Orfeo, M., Urbano, F., and Romeo, G.: Human a-fucosidase: Single residual enzymatic form in fucosidosis. Biochim. Bionhss. Acta, 429: 538 (1976).

5. Harris, H., and Watkins, J. F.: Hybrid cells derived from mouse and man: Artificial heterokaryons of mammalian cells from different species. Nature, 205: $640(1965)$.

6. Ho, M. W., Seck, J., Schmidt, D., Veath, M. L., Johnson, W., Brady, R. O.. and O'Brien, J. S.: Adult Gaucher's disease: Kindred studies and demonstration of a deficiency of acid $\beta$-glucosidiase in cultured fibroblasts. Amer. J. Hum. Genet.. 24: 37 (1972).

7. Kousseff, B. G., Beratis, N. G., Strauss, L.. Brill, P. W., Rosenficld, R. E., Kaplan, B., and Hirschhorn, K.: Fuconidosis type 2. Pediatries, 57: 205 (1976).

8. Loeb, H.. Tondeur, M., Jomniaux, G., Mockel-Pohl, S., and Vamos-Hurwitz. E.: Biochemical and ultrastructural studies in a case of mucopolysaccharidesis "F" (fucosidesis). Helv. Paediat. Acta, 36: 519 (1969).

9. Lowry, O. H., Rosebrough, N. J., Farr, A. L., and Randall, R. J.: Protein measurement with the Folin phenol reagent. J. Biol. Chem., 193: 26.5 (1951).

10. Matsuda, 1., Arashima, S., Anakura, M., Ege, A., and Hayata, 1.: Fucosidoxis. Tohoku 1 . Exp. Med., 109: 41 (1973).

11. Matsuda, I., Arashima, S., Anakura, M., and Oka, Y': $\alpha$-L-Fucosidase and $\alpha-$ D-mannosidase activity in the white blood cells in the disease and carrice state of fucosidosis. Clin. Chim. Acta, 48: 9 (1973).

12. Turner, B. M., Beratis, N. G., Turner, V. S., and llirschhorn, K.: Isozymes of human $\alpha$-L-fucosidase detectable by starch gel electrophoresis. Clin. Chim. Actia, 57: 29(1974).

13. Turner, B. M., Beratis, N. G., Turner, V.S., and Hirschhorn, K.: Silent allede as genetic basis of fucosidosis. Nature. 257: 391 (1975).

14. Turner, B. M., Turner, V. S., Beratis, N. G., and Hirschhorn, K.: Polymorphism of human $\alpha$ fucosidase. Amer. J. Hum. Genet., 27: 6.51 (1975).

15. Van Hoof, F., and Hers, H. G.: Mucopolysaccharidosis by absence of afucosidase. lancet, $i: 1198(1968)$.

16. Van Hoof, F., and llers, H. G.: The abnormalities of lysosomal enzymes in mucupolysaccharidoses. Europ. J. Biochem., 7: 34 (1968).

17. Zielke, K., Okada, S., and O'Brien, J.: Fucosidosis: Diagnosis by serum assay of $x$-L-fucosidase. J. Lab. Clin. Med., 79: 164 (1972).

18. Zielhe, K., Veath, M. L., and $O^{\prime}$ Brien, J. S.: Fucosidesis: Deficiency of alpha1.-fucosidase in cultured skin fibroblasts. J. Exp. Med., 1.36: 197 (1972).

19. Grand Island Biological Co., Grand Island, N.Y.

20. The authors wish to thank Dr. A. L. Miller of the University of California at 
San Diego, School of Medicine, Department of Neurosciences for the supply of the fucosidosis type 1 fibroblast strain.

21. Koch-Light Laboratories, Lid., Colnbrook, Buckinghamshire. England.

22. Sigma Chemical Co., Saint Louis, Mo.

23. Behringwerke, A. G., Matrburg-Lahn, Germany.

24. Connaught Laboratories, Lid., Toronto, Canada.

25. The studies on human subjects were performed with their informed consent or, in the cilse of children, with the informed consent of their parents.
26. This research was supported by United States Public Health Service Grant HD-02552 and Genetics Center Grant G.M-19443.

27. Requests for reprints should be addressed to: N. G. Beratis, M.D., Department of Pediatries, Division of Medical Genetics, Mount Sinai School of Medicine of the City University of New York, N. Y. 1(K)29 (USA).

28. Received for publication September 23, 1976.

29. Accepted for publication December 21, 1976 . 